International Journal of Pure and Applied Mathematics

Volume 107 No. 3 2016, 673-682

ISSN: 1311-8080 (printed version); ISSN: 1314-3395 (on-line version)

url: http://www.ijpam.eu

doi: 10.12732/ijpam.v107i3.15

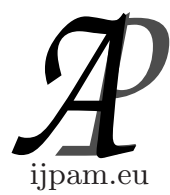

\title{
EDGE REGULAR PROPERTY OF COMPLEMENT AND $\mu$-COMPLEMENT OF A FUZZY GRAPH AND EDGE ADJACENCY SEQUENCE IN FUZZY GRAPH
}

\author{
K. Radha ${ }^{1}$, N. Kumaravel ${ }^{2} \S$ \\ ${ }^{1}$ P.G. Department of Mathematics \\ Periyar E.V.R. College \\ Tiruchirappalli, 620 023, Tamil Nadu, INDIA \\ ${ }^{2}$ Department of Mathematics \\ K.S.R. Institute for Engineering and Technology \\ Namakkal, 637 215, Tamil Nadu, INDIA
}

\begin{abstract}
In this paper, some theorems of edge regular fuzzy graphs are discussed with their complements and $\mu$-complements. A necessary and sufficient condition under which they are equivalent is provided. Finally, adjacency sequence of edges in a fuzzy graph is defined. Using the sequences, characterization for a fuzzy graph with at most four vertices to be edge regular is obtained.
\end{abstract}

AMS Subject Classification: 03E72, 05C72

Key Words: strong fuzzy graph, complete fuzzy graph, edge regular fuzzy graph, totally edge regular fuzzy graph, complement of a fuzzy graph and $\mu$-complement of a fuzzy graph

\section{Introduction}

Fuzzy graph theory was introduced by Azriel Rosenfeld in 1975 [8]. Though it is very young, it has been growing fast and has numerous applications in various fields. During the same time Yeh and Bang have also introduced various connectedness concepts in fuzzy graphs [10]. Mordeson (1994) introduced the

\footnotetext{
Received: January 17, 2016

Published: April 21, 2016

${ }^{\S}$ Correspondence author
}

(c) 2016 Academic Publications, Ltd.

url: www.acadpubl.eu 
concept of complement of fuzzy graphs [2]. M. S. Sunitha and A. Vijayakumar (2002) gave a modified definition of complement of fuzzy graph [9]. A. Nagoorgani and V. T. Chandrasekaran (2006), defined $\mu$-complement of a fuzzy graph [3], which is slightly different from the definition of complement of a fuzzy graph discussed by M. S. Sunitha and A. Vijayakumar. A. Nagoorgani and K. Radha defined the concept of incidence sequence of vertices in a fuzzy graph (2008) [6]. K. Radha and N. Kumaravel (2014) introduced the concept of edge regular fuzzy graphs [7]. In this paper, we provide some theorems on edge regular property of complements and $\mu$-complements of fuzzy graphs through various examples. We introduce edge adjacency sequence in a fuzzy graph and we use this sequence to study about the edge regular property of fuzzy graphs. First we go through some basic definitions in the next section.

\section{Basic Concepts}

Let $V$ be a non-empty finite set and $E \subseteq V \times V$. A fuzzy graph $G:(\sigma, \mu)$ is a pair of functions $\sigma: V \rightarrow[0,1]$ and $\mu: E \rightarrow[0,1]$ such that $\mu(x y) \leq \sigma(x) \wedge \sigma(y)$ for all $\mathrm{x}, \mathrm{y} \in \mathrm{V}$. Let $G:(\sigma, \mu)$ be a fuzzy graph on $G^{*}:(V, E)$. The degree of a vertex $x$ is $d_{G}(x)=\sum_{x \neq y} \mu(x y)$. The minimum degree of $G$ is $\delta(G)=$ $\wedge\left\{d_{G}(x), \forall x \in V\right\}$ and the maximum degree of $G$ is $\Delta(G)=\vee\left\{d_{G}(x), \forall x \in V\right\}$. Let $G:(\sigma, \mu)$ be a fuzzy graph on $G^{*}:(V, E)$. The total degree of a vertex $x$ is defined by $t d_{G}(x)=\sum_{x \neq y} \mu(x y)+\sigma(x)$.

Let $G:(\sigma, \mu)$ be a fuzzy graph on $G^{*}:(V, E)$. If each vertex in $G$ has same degree $k$, then $G$ is said to be a regular fuzzy graph or $k$-regular fuzzy graph. If each vertex in $G$ has same total degree $k$, then $G$ is said to be a totally regular fuzzy graph or $k$-totally regular fuzzy graph.

Let $G:(\sigma, \mu)$ be a fuzzy graph on $G^{*}:(V, E)$. The degree of an edge $x y \in E$ is

$$
d_{G}(x y)=\sum_{x \neq z} \mu(x z)+\sum_{z \neq y} \mu(z y)-2 \mu(x y) .
$$

The total degree of an edge $x y \in E$ is

$$
d_{G}(x y)=\sum_{x \neq z} \mu(x z)+\sum_{z \neq y} \mu(z y)-\mu(x y) .
$$

Let $G:(\sigma, \mu)$ be a fuzzy graph on $G^{*}:(V, E)$. If each edge in $G$ has same degree $k$, then $G$ is said to be an edge regular fuzzy graph or $k$-edge regular fuzzy graph. If each edge in $G$ has same total degree $k$, then $G$ is said to be a totally edge regular fuzzy graph or $k$-totally edge regular fuzzy graph. 
The order and size of a fuzzy graph $G$ are defined by $O(G)=\sum_{x \in V} \sigma(x)$ and $S(G)=\sum_{x y \in E} \mu(x y)$.

A fuzzy graph $G$ is strong, if $\mu(x y)=\sigma(x) \wedge \sigma(y)$ for all $x y \in E$. A fuzzy graph $G$ is complete, if $\mu(x y)=\sigma(x) \wedge \sigma(y)$ for all $x, y \in V$.

Let $G$ be a fuzzy graph. The complement of $G$ is defined as $\bar{G}:(\sigma, \bar{\mu})$, where

$$
\bar{\mu}(x y)=\sigma(x) \wedge \sigma(y)-\mu(x y),
$$

for all $x, y \in V$. The $\mu$-complement of $G$ is defined as $G^{\mu}:\left(\sigma, \mu^{\mu}\right)$, where

$$
\mu^{\mu}(x y)= \begin{cases}\sigma(x) \wedge \sigma(y)-\mu(x y), & \forall x y \in E, \\ 0, & \forall x y \notin E .\end{cases}
$$

The incidence sequence of a vertex $v$ in a fuzzy graph $G$ is defined as a sequence of membership values of edges incident to $v$ arranged in increasing order. It is denoted by $i s(v)$.

\section{Edge Regular and Totally Edge Regular Property of Complements}

Remark 1. If $G:(\sigma, \mu)$ is an edge regular fuzzy graph, then $\bar{G}:(\sigma, \bar{\mu})$ need not be edge regular fuzzy graph. For example, consider $G^{*}:(V, E)$ where $V=$ $\{a, b, c, d\}$ and $E=\{a b, b c, b d, c d, d a\}$. Define $G:(\sigma, \mu)$ by $\sigma(a)=0.9, \sigma(b)=$ $1, \sigma(c)=0.8, \sigma(d)=0.9$ and $\mu(a b)=0.4, \mu(b c)=0.5, \mu(b d)=0.9, \mu(c d)=$ $0.5, \mu(d a)=0.4$. Then $\bar{G}:(\sigma, \bar{\mu})$ is defined by $\bar{\mu}(a b)=0.5, \bar{\mu}(b c)=0.3, \bar{\mu}(a c)=$ $0.7, \bar{\mu}(c d)=0.3, \bar{\mu}(d a)=0.5$. Here $d_{G}(a b)=1.8$, for all $a b \in E$, but in $\bar{G}$ : $(\sigma, \bar{\mu}), d_{\bar{G}}(a b)=d_{\bar{G}}(b c)=d_{\bar{G}}(c d)=d_{\bar{G}}(d a)=1.5, d_{\bar{G}}(a c)=1.6$ and $t d_{\bar{G}}(a b)=$ $2, t d_{\bar{G}}(b c)=1.8, t d_{\bar{G}}(c d)=1.8, t d_{\bar{G}}(d a)=2, t d_{\bar{G}}(a c)=2.3$. Therefore $G:(\sigma, \mu)$ is 1 . 8 - edge regular fuzzy graph, but $\bar{G}:(\sigma, \bar{\mu})$ is neither edge regular fuzzy graph nor totally edge regular fuzzy graph.

Remark 2. If $G:(\sigma, \mu)$ is totally edge regular fuzzy graph, then $\bar{G}:(\sigma, \bar{\mu})$ need not be edge regular fuzzy graph. For example, consider $G^{*}:(V, E)$ where $V=\{a, b, c, d\}$ and $E=\{a b, a c, a d\}$. Define $G:(\sigma, \mu)$ by $\sigma(a)=$ $0.9, \sigma(b)=0.5, \sigma(c)=0.7, \sigma(d)=0.8$ and $\mu(a b)=0.5, \mu(a c)=0.4, \mu(a d)=0.7$. Then $\bar{G}:(\sigma, \bar{\mu})$ is defined by $\bar{\mu}(a c)=0.3, \bar{\mu}(a d)=0.1, \bar{\mu}(b c)=0.5, \bar{\mu}(b d)=$ $0.5, \bar{\mu}(c d)=0.7$. Here $t d_{G}(a b)=1.6$, for all $a b \in E$, but in $\bar{G}:(\sigma, \bar{\mu})$, $d_{\bar{G}}(a c)=1.3=d_{\bar{G}}(b d), d_{\bar{G}}(a d)=1.5=d_{\bar{G}}(b c), d_{\bar{G}}(c d)=1.4$ and $t d_{\bar{G}}(a c)=$ $1.6=t d_{\bar{G}}(a d), t d_{\bar{G}}(b c)=2, t d_{\bar{G}}(b d)=1.8, t d_{\bar{G}}(c d)=2.1$. Therefore $G:(\sigma, \mu)$ is 1 . 6 - totally edge regular fuzzy graph, but $\bar{G}:(\sigma, \bar{\mu})$ is neither edge regular fuzzy graph nor totally edge regular fuzzy graph. 
Theorem 3. Let $\mu(x, y)=[\sigma(x) \wedge \sigma(y)] / 2$, for all $x, y \in V$. Then $G:(\sigma, \mu)$ is a $k$ - edge regular fuzzy graph if and only if complement of $G:(\sigma, \mu)$ is also $k$ - edge regular.

Proof. Let

$$
\mu(x, y)=[\sigma(x) \wedge \sigma(y)] / 2, \text { for all } x, y \in V
$$

By the definition of complement, $\bar{\mu}(x, y)=\sigma(x) \wedge \sigma(y)-\mu(x u)$, for all $x, y \in V$. Therefore

$$
\bar{\mu}(x, y)=[\sigma(x) \wedge \sigma(y)] / 2,
$$

for all $x, y \in V[\mathrm{by}(1)]$. Hence

$$
\bar{\mu}(x, y)=\mu(x, y)
$$

Therefore

$$
d_{\bar{G}}(x y)=\sum_{x \neq z} \bar{\mu}(x z)+\sum_{z \neq y} \bar{\mu}(z y)-2 \bar{\mu}(x y) .
$$

By $(2), d_{G}(x y)=\sum_{x \neq z} \mu(x z)+\sum_{z \neq y} \mu(z y)-2 \mu(x y)$. Therefore $d_{\bar{G}(x y)}=$ $d_{G(x y)}$, for all $x y \in E$. Hence $G:(\sigma, \mu)$ is $k$ - edge regular fuzzy graph if and only if complement of $G:(\sigma, \mu)$ is also $k$ - edge regular.

Remark 4. The converse of theorem 3 need not be true.

For example, consider $G^{*}:(V, E)$, where

$$
V=\{a, b, c, d\}
$$

and

$$
E=\{a b, a c, a d, b c, b d, c d\} .
$$

Define $G:(\sigma, \mu)$ by $\sigma(a)=0.9, \sigma(b)=0.7, \sigma(c)=0.7, \sigma(d)=0.7$ and $\mu(a b)=$ $0.4, \mu(a c)=0.35, \mu(a d)=0.3, \mu(b c)=0.4, \mu(b d)=0.35, \mu(c d)=0.3$. Then $\bar{G}$ : $(\sigma, \bar{\mu})$ is defined by $\bar{\mu}(a b)=0.3, \bar{\mu}(a c)=0.35, \bar{\mu}(a d)=0.4, \bar{\mu}(b c)=0.3, \bar{\mu}(b d)=$ $0.35, \bar{\mu}(c d)=0.4$. Here $d_{G}(a b)=1.4$, for all $a b \in E$ and $d_{\bar{G}}(a b)=1.4$, for all $a b \in \bar{E}$. Therefore $G:(\sigma, \mu)$ and $\bar{G}:(\sigma, \bar{\mu})$ are edge regular fuzzy graphs, but $\mu(x, y) \neq[\sigma(x) \wedge \sigma(y)] / 2$, for $x, y \in V$.

Theorem 5. Let $\mu(x, y)=[\sigma(x) \wedge \sigma(y)] / 2$, for all $x, y \in V$. Then $G:(\sigma, \mu)$ is a $k$ - totally edge regular fuzzy graph if and only if complement of $G:(\sigma, \mu)$ is also $k$ - totally edge regular.

Proof. Proof is similar to proof of theorem 3. 
Theorem 6. Let $G:(\sigma, \mu)$ be a fuzzy graph on a regular graph $G^{*}:(V, E)$ such that $\mu$ is a constant function. Then $G:(\sigma, \mu)$ and $\bar{G}:(\sigma, \bar{\mu})$ are edge regular fuzzy graphs.

Proof. Let $\mu(e)=c$, for all $e \in E$, where $c$ is a constant. Let $G^{*}$ be $k$ regular. Then $d_{G^{*}}(x)=k$, for all $x \in V$, where $k$ is a constant. First let us prove that $G:(\sigma, \mu)$ is an edge regular fuzzy graph. By the definition of edge degree, $d_{G}(x y)=\sum_{x \neq z} \mu(x z)+\sum_{z \neq y} \mu(z y)-2 \mu(x y)$. Therefore

$$
d_{G}(x y)=\sum_{x \neq z} c+\sum_{z \neq y} c-2 c=c d_{G^{*}}(x)+c d_{G^{*}}(y)-2 c
$$

or $d_{G}(x y)=c k+c k-2 c=2 c(k-1)$. Hence $G:(\sigma, \mu)$ is an edge regular fuzzy graph. Next we prove that $\bar{G}:(\sigma, \bar{\mu})$ is an edge regular fuzzy graph. Let $|V|=n$. Then $\bar{G}^{*}$ is $(n-1-k)$ - regular. By the definition of complement, $\bar{\mu}(x y)=\sigma(x) \wedge \sigma(y)-\mu(x y)$, for all $x, y \in V$,

$$
\bar{\mu}(x y)= \begin{cases}c \wedge c-c, & \forall x y \in E \\ c \wedge c, & \text { otherwise. }\end{cases}
$$

Therefore $\bar{\mu}(x y)=c$, for all $x y \in \bar{E}$. By the definition of edge degree, $d_{\bar{G}}(x y)=$ $\sum_{x \neq z} \bar{\mu}(x z)+\sum_{z \neq y} \bar{\mu}(z y)-2 \bar{\mu}(x y)$, for all $x y \in \bar{E}$. Therefore

$$
d_{\bar{G}}(x y)=\sum_{x \neq z} c+\sum_{z \neq y} c-2 c=c d_{\bar{G}^{*}}(x)+c d_{\bar{G}^{*}}(y)-2 c .
$$

Hence $d_{\bar{G}}(x y)=2 c(n-k-2)$, for all $x y \in \bar{E}$. Hence $\bar{G}:(\sigma, \bar{\mu})$ is an edge regular fuzzy graph.

Corollary 7. Let $G:(\sigma, \mu)$ be a strong fuzzy graph such that $\sigma$ is a constant function and let $G^{*}$ be a regular graph. Then $G:(\sigma, \mu)$ and $\bar{G}:(\sigma, \bar{\mu})$ are edge regular fuzzy graphs.

Proof. Given $G:(\sigma, \mu)$ is a strong fuzzy graph such that $\sigma$ is a constant function. Then $\mu$ is a constant function. Therefore the result follows from theorem 6 .

Remark 8. The converse part of theorem 6 and corollary 7 need not be true. It can be seen from the following example. Consider $G^{*}:(V, E)$ where $V=\{a, b, c, d\}$ and $E=\{a b, a c, b c, c d\}$. Define $G:(\sigma, \mu)$ by $\sigma(a)=0.4, \sigma(b)=$ $0.6, \sigma(c)=0.4, \sigma(d)=0.4$ and $\mu(a b)=0.2, \mu(a c)=0.4, \mu(b c)=0.4, \mu(c d)=$ 
0.2. Then $\bar{G}:(\sigma, \bar{\mu})$ is defined by $\bar{\mu}(a b)=0.2, \bar{\mu}(a d)=0.4, \bar{\mu}(b d)=0.4, \bar{\mu}(c d)=$ 0.2. Here $d_{G}(a b)=0.8$, for all $a b \in E$ and $d_{\bar{G}}(a b)=0.8$, for all $a b \in \bar{E}$. Therefore $G:(\sigma, \mu)$ and $\bar{G}:(\sigma, \bar{\mu})$ are edge regular fuzzy graphs, but $\sigma$ and $\mu$ are not constant functions, $G:(\sigma, \mu)$ is not a strong fuzzy graph and $G^{*}:(V, E)$ is not a regular graph.

\section{Edge Regular and Totally Edge Regular Property of $\mu$-Complements}

Result 9. When $G^{*}$ is complete, the complement of a fuzzy graph $G$ is same as the $\mu$-complement of $G$.

Proof. By the definition

$$
\mu^{\mu}(x y)= \begin{cases}\sigma(x) \wedge \sigma(y)-\mu(x y), & \text { if } \mu(x y)>0 \\ 0, & \text { if } \mu(x y)=0\end{cases}
$$

Therefore $\mu^{\mu}(x y)=\sigma(x)+\sigma(y)-\mu(x y)$, for all $x, y \in V$. Hence $\mu^{\mu}(x y)=$ $\bar{\mu}(x y)$,forall $x, y \in V$ and $x y \in E$. Also $\bar{\sigma}(x)=\sigma(x)=\sigma^{\mu}(x)$, for all $x \in V$. Hence the result.

Remark 10. If $G:(\sigma, \mu)$ is an edge regular fuzzy graph, then $G^{\mu}:\left(\sigma, \mu^{\mu}\right)$ need not be edge regular fuzzy graph. For example, consider $G^{*}:(V, E)$ where $V=\{a, b, c, d\}$ and $E=\{a b, a d, b c, b d, c d\}$. Define $G:(\sigma, \mu)$ by $\sigma(a)=$ $0.9, \sigma(b)=1, \sigma(c)=0.8, \sigma(d)=1$ and $\mu(a b)=0.4, \mu(a d)=0.4, \mu(b c)=$ $0.5, \mu(b d)=0.9, \mu(c d)=0.5$. Then $G^{\mu}:\left(\sigma, \mu^{\mu}\right)$ is defined by $\mu^{\mu}(a b)=$ $0.5, \mu^{\mu}(a d)=0.5, \mu^{\mu}(b c)=0.3, \mu^{\mu}(b d)=0.1, \mu^{\mu}(c d)=0.3$. Here $d_{G}(a b)=1.8$, for all $a b \in E, d_{G^{\mu}}(a b)=d_{G^{\mu}}(a d)=d_{G^{\mu}}(b c)=d_{G^{\mu}}(c d)=0.9, d_{G^{\mu}}(b d)=1.6$ and $t d_{G^{\mu}}(a b)=1.4=t d_{G^{\mu}}(a d), t d_{G^{\mu}}(b c)=1.2=t d_{G^{\mu}}(c d), t d_{G^{\mu}}(b d)=1.7$. Therefore $G:(\sigma, \mu)$ is 1 . 8 - edge regular fuzzy graph, but $G^{\mu}:\left(\sigma, \mu^{\mu}\right)$ is neither edge regular fuzzy graph nor totally edge regular fuzzy graph.

Remark 11. If $G:(\sigma, \mu)$ is totally edge regular fuzzy graph, then $G^{\mu}$ : $\left(\sigma, \mu^{\mu}\right)$ need not be edge regular fuzzy graph. For example, consider $G^{*}$ : $(V, E)$ where $V=\{a, b, c, d, e, f\}$ and $E=\{a b, b c, c d, d e, e f, f a\}$. Define $G$ : $(\sigma, \mu)$ by $\sigma(a)=0.9, \sigma(b)=0.6, \sigma(c)=0.5, \sigma(d)=0.7, \sigma(e)=0.6, \sigma(f)=0.7$ and $\mu(a b)=0.6, \mu(b c)=0.4, \mu(c d)=0.3, \mu(d e)=0.6, \mu(e f)=0.4, \mu(f a)=$ 0.3. Then $G^{\mu}:\left(\sigma, \mu^{\mu}\right)$ is defined by $\mu^{\mu}(b c)=0.1, \mu^{\mu}(c d)=0.2, \mu^{\mu}(e f)=$ $0.2, \mu^{\mu}(f a)=0.4$. Here $t d_{G}(a b)=1.3$, for all $a b \in E, d_{G^{\mu}}(b c)=0.2, d_{G^{\mu}}(c d)=$ $0.1, d_{G^{\mu}}(e f)=0.4, d_{G^{\mu}}(f a)=0.2$ and $t d_{G^{\mu}}(b c)=0.3=t d_{G^{\mu}}(c d), t d_{G^{\mu}}(e f)=$ 
$0.6=t d_{G^{\mu}}(f a)$. Therefore $G:(\sigma, \mu)$ is 1.3 - totally edge regular fuzzy graph, but $G^{\mu}:\left(\sigma, \mu^{\mu}\right)$ is neither edge regular fuzzy graph nor totally edge regular fuzzy graph.

Theorem 12. Let $\mu(x y)=[\sigma(x) \wedge \sigma(y)] / 2$, for all $x y \in E$. Then $G:(\sigma, \mu)$ is a $k$ - edge regular fuzzy graph if and only if $G^{\mu}:\left(\sigma, \mu^{\mu}\right)$ is also $k$ - edge regular.

Proof. Given

$$
\mu(x y)=[\sigma(x) \wedge \sigma(y)] / 2, \text { for all } x y \in E .
$$

By the definition of $\mu$ - complement

$$
\mu^{\mu}(x y)= \begin{cases}\sigma(x) \wedge \sigma(y)-\mu(x y), & \text { if } \mu(x y)>0 \\ 0, & \text { if } \mu(x y)=0\end{cases}
$$

Therefore $\mu^{\mu}(x y)=\sigma(x) \wedge \sigma(y)-[\sigma(x) \wedge \sigma(y)] / 2$, for all $x y \in E$ (see (3)), or

$$
\mu^{\mu}(x y)=[\sigma(x) \wedge \sigma(y)] / 2
$$

for all $x y \in E$. Hence

$$
\begin{aligned}
d_{G^{\mu}}(x y)=\sum_{x \neq z} \mu^{\mu}(x z)+\sum_{z \neq y} \mu^{\mu}(z y)-2 \mu^{\mu}(x y) & \\
& =\sum_{x \neq z} \mu(x z)+\sum_{z \neq y} \mu(z y)-2 \mu(x y)=d_{G}(x y),
\end{aligned}
$$

for all $x y \in E$. Hence $G:(\sigma, \mu)$ is a $k$ - edge regular fuzzy graph if and only if $G^{\mu}:\left(\sigma, \mu^{\mu}\right)$ is also $k$ - edge regular.

Remark 13. The converse part of theorem 12 need not be true. In the following example, $G:(\sigma, \mu)$ and $G^{\mu}:\left(\sigma, \mu^{\mu}\right)$ are edge regular, but $\mu(x y) \neq$ $[\sigma(x) \wedge \sigma(y)] / 2$, for all $x y \in E$. Consider $G^{*}:(V, E)$ where $V=\{a, b, c, d\}$ and $E=\{a b, b c, c d, d a\}$. Define $G:(\sigma, \mu)$ by $\sigma(a)=0.9, \sigma(b)=0.7, \sigma(c)=$ $0.8, \sigma(d)=0.7$ and $\mu(a b)=0.4, \mu(b c)=0.4, \mu(c d)=0.3, \mu(d a)=0.3$. Then $G^{\mu}:\left(\sigma, \mu^{\mu}\right)$ is defined by $\mu^{\mu}(a b)=0.3, \mu^{\mu}(b c)=0.3, \mu^{\mu}(c d)=0.4, \mu^{\mu}(d a)=0.4$. Here $d_{G}(a b)=0.7=d_{G^{\mu}}(a b)$, for all $a b \in E$.

Theorem 14. Let $\mu(x y)=[\sigma(x) \wedge \sigma(y)] / 2$, for all $x y \in E$. Then $G:(\sigma, \mu)$ is a $k$ - totally edge regular fuzzy graph if and only if $G^{\mu}:\left(\sigma, \mu^{\mu}\right)$ is also $k$ totally edge regular.

Proof. Proof is similar to proof of theorem 12. 


\section{Edge Adjacency Sequences}

Definition 15. The adjacency sequence of an edge $e$ in a fuzzy graph $G$ is defined as a sequence of membership values of edges adjacent to $e$ arranged in increasing order. It is denoted by as $(e)$.

Remark 16. 1. The number of elements in as $(e)$ is the degree of $e$ in $G^{*}$.

2. The sum of all elements in $a s(e)$ is the degree of $e$ in $G$.

Example 17. Consider the fuzzy graph in the following example.

Consider $G^{*}:(V, E)$, where $V=\{a, b, c, d\}$ and $E=\{a b, a d, b d, c d\}$. Define $G:(\sigma, \mu)$ by $\sigma(a)=0.6, \sigma(b)=0.7, \sigma(c)=0.8, \sigma(d)=0.5$ and $\mu(a b)=$ $0.4, \mu(a d)=0.5, \mu(b d)=0.6, \mu(c d)=0.5$. The adjacency sequences of the edges are $a s(a b)=(0.5,0.6), a s(a d)=(0.4,0.5,0.6), a s(b d)=(0.4,0.5,0.5), a s(c d)=$ $(0.5,0.6)$.

Remark 18. When $G$ is edge regular, the underlying graph $G^{*}$ need not be edge regular. For example, consider $G^{*}:(V, E)$ where $V=\{a, b, c, d\}$ and $E=\{a b, a d, b d, c d\}$. Define $G:(\sigma, \mu)$ by $\sigma(a)=0.4, \sigma(b)=0.7, \sigma(c)=$ $0.6, \sigma(d)=0.5$ and $\mu(a b)=0.2, \mu(a d)=0.4, \mu(b d)=0.4, \mu(c d)=0.2$. This is an edge regular fuzzy graph of degree 0.8 whose underlying graph is not edge regular. Also $a s(a b) \neq a s(b d)$. Hence in an edge regular fuzzy graph, all the edges need not have the same adjacency sequence.

Remark 19. Even if both $G$ and $G^{*}$ are edge regular, then all the edges need not have the same adjacency sequence. For example, consider $G^{*}:(V, E)$ where $V=\{a, b, c, d\}$ and $E=\{a b, a c, a d, b c, b d, c d\}$. Define $G:(\sigma, \mu)$ by $\sigma(a)=0.4, \sigma(b)=0.7, \sigma(c)=0.6, \sigma(d)=0.5$ and $\mu(a b)=0.3, \mu(a c)=$ $0.2, \mu(a d)=0.2, \mu(b c)=0.2, \mu(b d)=0.2, \mu(c d)=0.1$. Here both $G$ and $G^{*}$ are edge regular. But $a s(a d) \neq a s(c d)$.

Theorem 20. If $G^{*}$ is a triangle, the degree of an edge in $G$ is the degree of the vertex opposite to it in $G$.

Proof. Consider the fuzzy graph $G$ on a triangle $G^{*}$. Let the vertices of $G$ be $a, b$ and $c$. In $G$, opposite edge of the vertex $a$ is $b c$, opposite edge of the vertex $b$ is $c a$ and opposite edge of the vertex $c$ is $a b$. Then $d_{G}(a)=\mu(a b)+\mu(a c)=$ $d_{G}(b c), d_{G}(b)=\mu(b c)+\mu(b a)=d_{G}(c a)$ and $d_{G}(c)=\mu(c a)+\mu(c b)=d_{G}(a b)$. Hence the degree of an edge in $G$ is the degree of the vertex opposite to it in G.

Theorem 21. Let $G^{*}$ be a triangle. Then $G$ is an edge regular fuzzy graph if and only if $\mu$ is a constant and all the edges have the same adjacency 
sequence.

Proof. Let $G^{*}$ be a triangle. Then $G^{*}$ is 2 - regular and 2 - edge regular. Assume that $\mu$ is a constant and all the edges have the same adjacency sequence. Then $G$ is an edge regular fuzzy graph.

Conversely, assume that $G$ is $k$ - edge regular fuzzy graph. Let the vertices of $G$ be $v_{1}, v_{2}$ and $v_{3}$. Let the membership values of the three edges $v_{1} v_{2}, v_{2} v_{3}$ and $v_{3} v_{1}$ be $k_{1}, k_{2}, k_{3}$ respectively, where $0 \leq k_{i} \leq 1, i=1,2,3$.

Since $d\left(v_{i} v_{j}\right)=k, i, j=1,2,3$ and $i \neq j$, we have

$$
\begin{aligned}
& k_{1}+k_{2}=k, \\
& k_{1}+k_{3}=k, \\
& k_{2}+k_{3}=k .
\end{aligned}
$$

Equations (4)-(5) imply $k_{2}-k_{3}=0 \Rightarrow k_{2}=k_{3}$.

Equations (5)-(6) imply $k_{1}-k_{2}=0 \Rightarrow k_{1}=k_{2}$.

Hence $k_{1}=k_{2}=k_{3}$. Substituting $k_{1}=k_{2}$ in (4), we get $k_{1}=k / 2$.

Therefore $k_{1}=k_{2}=k_{3}=k / 2$. Hence each edge has the same adjacency sequence $(k / 2, k / 2)$ and $\mu$ is a constant.

Theorem 22. Let $G^{*}$ be a complete graph on four vertices. Then $G$ is $k$ - edge regular if and only if sum of the membership values of edges in each 1 factor is $k / 2$.

Proof. Let $G^{*}$ be a complete graph on four vertices. Then $G^{*}$ is 4 - edge regular and every edge in $G^{*}$ is adjacent to two 1 - factors. Assume that sum of the membership values of edges in each 1 - factor is $k / 2$. Then $G$ is $k$ edge regular. Conversely, assume that $G$ is $k$ - edge regular fuzzy graph. To prove that sum of the membership values of edges in each 1 - factor is $k / 2$. Let the vertices of $G$ be $v_{1}, v_{2}, v_{3}$ and $v_{4}$. Let the membership values of the six edges $v_{1} v_{2}, v_{2} v_{3}, v_{3} v_{4}, v_{4} v_{1}, v_{1} v_{3}$ and $v_{2} v_{4}$ be $k_{1}, k_{2}, k_{3}, k_{4}, k_{5}, k_{6}$ respectively, where $0 \leq k_{i} \leq 1, i=1,2,3,4,5,6$. Since $G$ is $k$ - edge regular fuzzy graph, $d\left(v_{i} v_{j}\right)=k, i, j=1,2,3,4$ and $i \neq j$. Hence, we have

$$
\begin{aligned}
& k_{4}+k_{5}+k_{6}+k_{2}=k, \\
& k_{1}+k_{5}+k_{6}+k_{3}=k, \\
& k_{4}+k_{5}+k_{6}+k_{2}=k, \\
& k_{1}+k_{5}+k_{6}+k_{3}=k, \\
& k_{1}+k_{4}+k_{2}+k_{3}=k, \\
& k_{1}+k_{2}+k_{3}+k_{4}=k .
\end{aligned}
$$


Equations (7)-(8) imply $k_{2}+k_{4}-k_{1}-k_{3}=0 \Rightarrow k_{2}+k_{4}=k_{1}+k_{3}$. Using this fact in (11), we receive $k_{2}+k_{4}=k / 2=k_{1}+k_{3}$.

Equations (9)-(11) imply $k_{5}+k_{6}-k_{1}-k_{3}=0 \Rightarrow k_{5}+k_{6}=k_{1}+k_{3}$ $\Rightarrow k_{5}+k_{6}=k / 2$.

Therefore $k_{1}+k_{3}=k_{2}+k_{4}=k_{5}+k_{6}=k / 2$. Hence sum of the membership values of edges in each 1 - factor is $k / 2$.

\section{References}

[1] Frank Harary, Graph Thoery, Narosa /Addison Wesley, Indian Student Edition, India (1988).

[2] J. N. Mordeson, C. S. Peng, Operations on Fuzzy Graphs, Information Sciences, Volume 79 (1994), $159-170$.

[3] A. Nagoorgani, V. T. Chandrasekaran, Free Nodes and Busy Nodes of a Fuzzy Graph, East Asian mathematical journal, Volume 22, Number 2 (2006), 163 - 170.

[4] A. Nagoorgani, J. Malarvizhi, Properties of $\mu$-Complement of a Fuzzy Graph, International Journal of Algorithms, Computing and Mathematics, Volume 2, Number 3 (2009), $73-83$.

[5] A. Nagoorgani, K. Radha, On Regular Fuzzy Graphs, Journal of Physical Sciences, Volume 12 (2008), 33 - 40.

[6] A. Nagoorgani, K. Radha, Some Sequences in Fuzzy Graphs, Far and East Journal of Applied Mathematics, Volume 31, Number 3 (2008), 321 - 335.

[7] K. Radha, N. Kumaravel, On Edge Regular Fuzzy Graphs, International Journal of Mathematical Archive, Volume 5, Issue 9 (2014), 100 - 112.

[8] A. Rosenfeld, Fuzzy graphs, in: L. A. Zadeh, K. S. Fu, K. Tanaka and M. Shimura, (editors), Fuzzy sets and their applications to cognitive and decision process, Academic press, New York (1975), 77 - 95.

[9] M. S. Sunitha, A. Vijayakumar, Complement of a Fuzzy Graph, Indian Journal of Pure and Applied Mathematics, volume 33, Issue 9 (2002), 1451 - 1464.

[10] R. T. Yeh and S. Y. Bang, Fuzzy relations, fuzzy graphs, and their applications to clustering analysis, in: L. A. Zadeh, K. S. Fu, K. Tanaka and M. Shimura, (editors), Fuzzy sets and their applications to cognitive and decision process, Academic press, New York (1975), $125-149$. 